\title{
Swathed Ureter, an Enigma in Diagnosis- A Pictorial Essay
}

\author{
Kareem, Abdul M.M*, A. Riaz Ahmed \\ Department of Radiology, School of Medicine, Taylor's University, Malaysia
}

*Corresponding author: Abdul Kareem Meera Mohaideen, Department of Radiology, School of Medicine, Taylor's University, No.1Jalan Taylor's, 47500-Subang Jaya, Selangor, Malaysia

\begin{abstract}
This pictorial essay is an educational article aiming to provide both textual and visual portrayals consisting of a collection of images and texts on an important issue by reviewing and extrinsic encasing pathology of the ureter to provide a guide to those who are involved in diagnostic intervention. While considering diseases of the urinary system, physicians mainly focus on the kidneys and the bladder. Only scant attention is paid to the ureters. Most of the UTO due to calculi are readily identifiable whereas many cases of ureteric exterior encasement are frequently missed from early detection even by experienced clinicians and radiologists. Failure in recognition of the encasement of ureters and its causes may lead to mistaken diagnosis with resultant inappropriate management. However, problems with the ureters can adversely affect the functioning of the kidneys and could even be lethal. In this article we focus only 'encasement of ureters' with a few common examples and salient signs that help in the diagnosis.
\end{abstract}

Keywords: Encasement of Ureters; Endometriosis; MRI; RPF; Obstructive Uropathy

\section{Background}

Urinary tract Obstruction (UTO) an alarming but common clinical condition affecting more women than men at any age, though common in 20 to 60 years of age having an overall incidence of hydronephrosis in $3.1 \%$ of autopsy. Over time, UTO results in irreversible loss of numerous nephrons leading to obstructive nephropathy and end-stage renal failure. If the obstruction of the ureter is partial and brief and if intervention is done at correct time, after relief of obstruction. complete recovery of renal function is possible. One has to be aware that UTO lasting more than 24 hours may cause irreversible loss of renal function. Radiology investigations may show UTO without ureteric dilation and dilation of ureter without UTO creating potential pitfall in radiologic diagnosis of UTO. Most of the UTO due to calculi are readily identifiable whereas many cases of ureteric exterior encasement are frequently missed from early detection even by experienced clinicians and radiologists. Failure in recognition of the encasement of ureters and causes may lead to mistaken diagnosis with resultant inappropriate management [1]. The most common benign cause of encasement of ureters is retroperitoneal fibrosis and the most frequent malignant causes are extension from an adjacent primary tumour such as sarcoma, lymphoma, (E.g.: sarcomas and lymphomas of uterus, ovaries, urinary bladder and prostate). Among benign conditions of swathed ureters, Extrinsic benign tumours, Retroperitoneal lymphadenopathy, Retroperitoneal abscess, Retroperitoneal fibrosis, Inflammatory abdominal aortic aneurysm or iliac artery aneurysm, and Endometriosis are significant. Chronic fibrosing conditions of the abdomen may involve multiple systems by their proliferative deep fibromatoses which form pseudotumor which cannot be differentiated from neoplastic conditions at imaging. Peri-ureteral inflammation (E.g.: peritonitis, salpingitis, and diverticulitis), multifocal idiopathic fibrosclerosis and schistosomiasis are some other known causes. Encasement may also be associated with blocked ureter [2-4]. Besides the inherent features of the disease causing the encasement of the ureters, in general, clinical features include recurrent fever, pain abdomen, oliguria, frequency, dysuria, haematuria nocturia, and hypertension. Patients may also present with fatigue, anorexia, weight loss, fever, hydroceles, scrotal pain, lower extremity oedema, and pulmonary embolism. Since ureters may be affected, various degrees of ureteral obstruction, hydronephrosis, and renal failure are also considered early and common clinical manifestations. In this article, we will review the four important diseases that cause ureteric encasement with some key imaging features for the diagnosis [5,6].

\section{Imaging}

On imaging ureteral pathology, plain abdominal radiography does not play a major role in imaging. Intravenous excretory 
urography (EUG or IVU) was once the study of choice for evaluating the renal collecting system and ureters; although now replaced by Computed Tomography urography (CTU), IVU is still performed in some centres. IVU has limited utility in patients with impaired renal function. In patients with renal insufficiency the risk for contrast-induced nephropathy from iodinated contrast media is high [7-10]. Systemic IV contrast should be avoided, and direct injection of a contrast medium can be performed with antegrade or retrograde pyelography. This allows evaluation of the collecting system and ureters and the opportunity for interventions such as stent placement. In cases where a detectable mass is not present, computed tomography and ultrasound, while helpful, are probably less sensitive and less specific than the retrograde ureterogram. Ultrasonography is not at all used to evaluate mid ureteral stricture or encasement; but is useful to diagnose if ureters are well distended in urinary obstruction due to a retroperitoneal mass and retroperitoneal fibrosis. However, overlying bowel often obscures visualization of the mid-ureter.

CT scan is the first choice to demonstrate ureters CT urography (CTU) is the major imaging modality for evaluating the ureters and secondary findings that help to narrow the differential diagnosis of the cause of the ureteral stricture and allows visualization of adjacent structures to differentiate an extrinsic pathology from an intrinsic process. For CTU, a three-scan CT protocol is used. After voiding completely all patients are asked to drink $800-1000 \mathrm{~mL}$ of water immediately prior to the examination [11]. The urinary system is imaged in both unenhanced and contrast enhanced CT (CECT) scan, by using multidetector helical CT scanner. On CECT, $100 \mathrm{~mL}$ of Non-ionic Low-osmolar contrast media (LOCM) is injected $(2.5 \mathrm{ml} / \mathrm{sec})$; the kidneys are scanned $25-80$ seconds after intravenous administration of the contrast for cortico-medullary phase, after 100 seconds for nephrographic phase, 8-10 minutes after contrasting medium injection for pyelographic phase, by using a maximum collimation of $1.0 \mathrm{~mm}$. All three scans are performed with a tube voltage of $120 \mathrm{kVp}$ and a tube current of $200 \mathrm{mAs}$.

Magnetic resonance urography (MRU) is very useful in patients with renal failure and or with Iodine contrast allergy. Absence of ionizing radiation, evaluation of paediatric patients and pregnant women, the high T2 signal intensity of urine in the dilated collecting system, are the advantages. Disadvantages of MRU are higher cost, motion artifacts from respiration and ureteral peristalsis, small field of view because of coil size nephrogenic systemic fibrosis secondary to gadolinium and bowel artifacts, However, when compared with CT, MRI offers better contrast resolution, but CT has higher spatial resolution [12-14]. Among Nuclear Medicine imaging in the setting of ureteral stricture and obstruction, diuretic renograms can be used to differentiate collecting system dilation from urinary obstruction. Radionuclide studies are not used for the detection of encasement of the ureters although positron emission tomography (PET) scan may show increased uptake. The role of PET in evaluating urothelial lesions is limited. Occasionally, ureteral obstruction may be identified in patients undergoing PET for other processes $[15,16]$. In general, on imaging, fibrosis displays hypoechogenic with acoustic shadowing at ultrasonography (US), hypovascularity at Doppler imaging, isoattenuating to muscle at computed tomography (CT), and isointensity at T1W1 and markedly low signal intensity at T2-weighted imaging. Due to reduced cellularity, the fibrous tissue typically does not show significant restriction at diffusion-weighted imaging. Contrastenhanced CT and MR will not be informative although delayed phase enhancement may be present. Other diseases mimicking Chronic fibrosing conditions such as mesenteric panniculitis, retractile mesenteritis, mesenteric carcinoid, Crohn disease with fibrofatty proliferation, and desmoid (mesenteric fibromatosis), carcinoid and carcinomatosis, mesenteric lipogenic liposarcoma will be the primary differential diagnoses [17].

\section{IgG4-related sclerosing disorders}

Retroperitoneal Fibrosis (RPF) is now considered an important abdominal component of the group of IgG4-related sclerosing disorders. It is an aggressive, rare fibro- proliferative process causing deposition of unencapsulated fibrous tissue masses in the retroperitoneal space usually centered at the lumbosacral junction. it is typically seen in men (2-3 times commoner than in women) between the 5 th and 7 th decades of life. Up to $15 \%$ of RPF patients have additional fibrotic processes in the mediastinum, thyroid (Riedel thyroiditis), biliary tree. sclerosing mesentery, and orbital tissue(pseudotumor). It leads to progressive encasement of the retroperitoneal structures, especially the ureters. and may cause hydronephrosis secondary to the extrinsic compression of the ureters [18]. RPF plaque may involve both ureters and may draw them medially towards the spine. This appearance on retrograde pyelogram resembles a narrow-waisted maiden (Figure 1a). Encasement of the ureter cause abrupt tapered narrowing of the ureteral lumen The dilated proximal ureter (the bullet) and the nondilated, encased distal ureter may appear as 'The bullet and bodkin' and this appearance can be caused by malignancy such as lymphoma, or rarely by RPF (Figure 1b). However, on the above imaging pictures, the ureteric encasement is not visually demonstrable. On CT, the area of fibrosis and encasement appears as a soft-tissue mass with variable contrast enhancement. The active and inactive stages of the disease can be differentiated on CT and MRI. In the retroperitoneum, RPF tissue surrounds the aorta (arrow) sparing posterior aspect (Figure 2a) indicating a benign stage. In contrast to the figure above, in malignant RPF, the RPF has mass effect with adjacent structures (Figure $2 \mathrm{~b}$ below). Another noteworthy finding is the intensity difference of RPF mass on MRI imaging (Figure 3). A high intensity signal of the mass on T2WI is suggestive of early active RPF whereas a low intensity signal on T2WI is highly suggestive of benign RPF in a late inactive stage. On FDG PET/CT imaging, the fibrous tissue in the affected areas exhibit avid FDG uptake (Figure 4b). When the infiltrating tissue encompass both ureters and compromise the lumen, bilateral hydronephrosis will ensue regardless of the location of the mass in the abdomen or pelvis (Figure 5a-b). Abdominal pain and obstructive uropathy 
should, therefore, raise suspicion for retroperitoneal fibrosis and imaging should be obtained. On the other hand, new onset of hypertension, flank pain radiating to groin in a known case of RPF or malignancy should arise the suspicion of ureteral encasement and appropriate imaging should confirm [19]. Failure in recognition of the encasement of ureters and causes may lead to mistaken diagnosis with resultant inappropriate management.

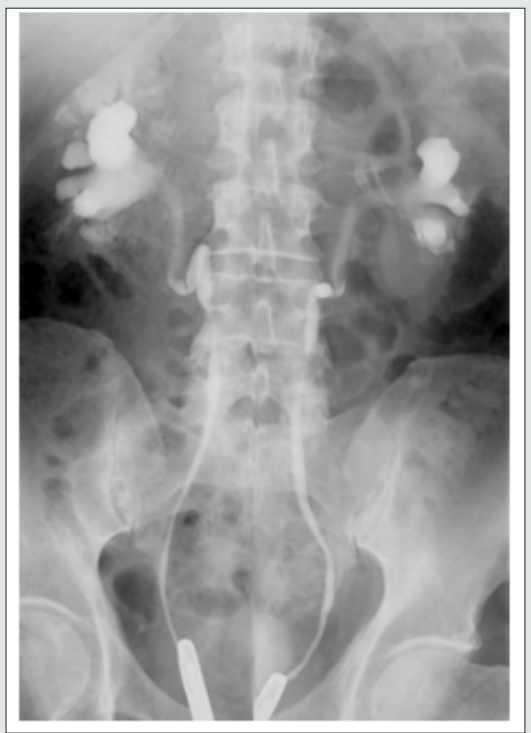

Figure 1: Retrograde ureteropyelogram 1a. Maiden waist deformity.

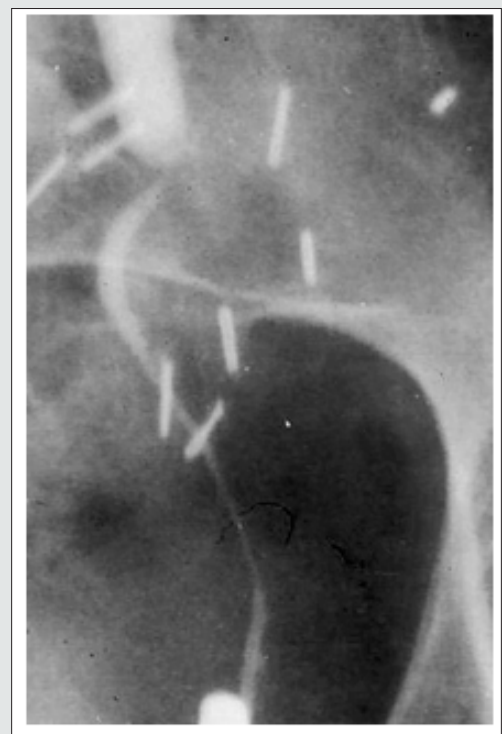

Figure 1b: Bullet and Bodkin Sign (Courtesy: Classic Signs in Uroradiology; Raymond B. Dyer, Michael, Ronald) 


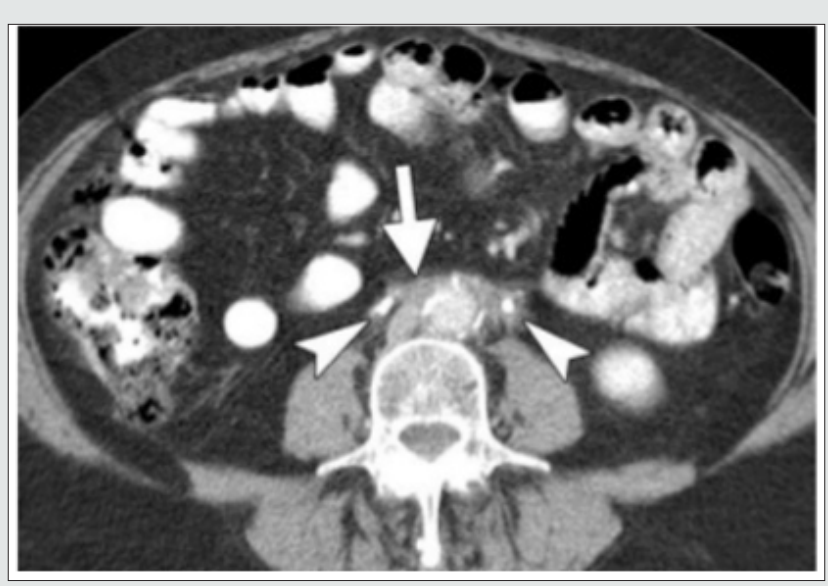

Figure 2a: Plaque-like Soft tissue surrounding the aorta (arrow) sparing posterior aspect without elevating the aorta from the spine; encases both ureters (arrow heads) findings indicative of benign RPF.

(courtesy: Ali Jiwani https://www.slideshare.net/AliJiwani/retroperitoneal-fibrosis- radiology)



Figure 2b: Axial CECT demonstrates well defined mass surrounding Aorta and IVC pushing the aorta anterior to the spine, a finding suggestive of malignant RPF.

(courtesy: Ali Jiwani https://www.slideshare.net/Alijiwani/retroperitoneal-fibrosis-radiology)

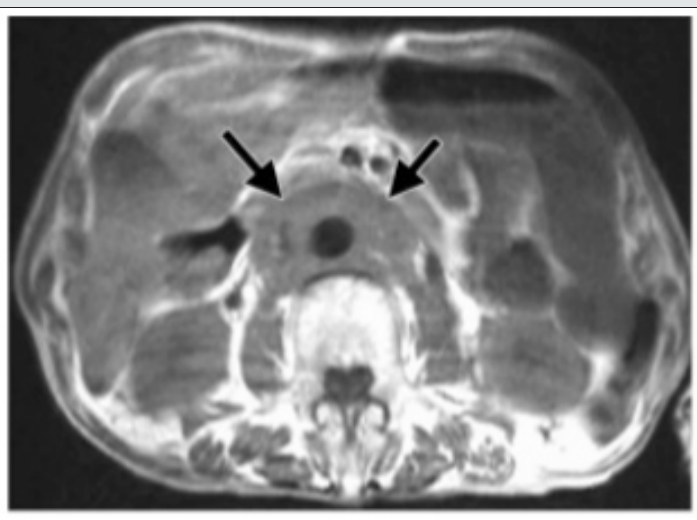

a.



b.

Figure 3a: Histologically confirmed idiopathic RPF mass surrounding the abdominal aorta and IVC, showing medium intensity on T1WI (3a); and low signal intensity on T2WI (3b) a finding suggestive of late stage. (courtesy: Ali Jiwani https://www. slideshare.net/ Alijiwani/retroperitoneal-fibrosis- radiology) 

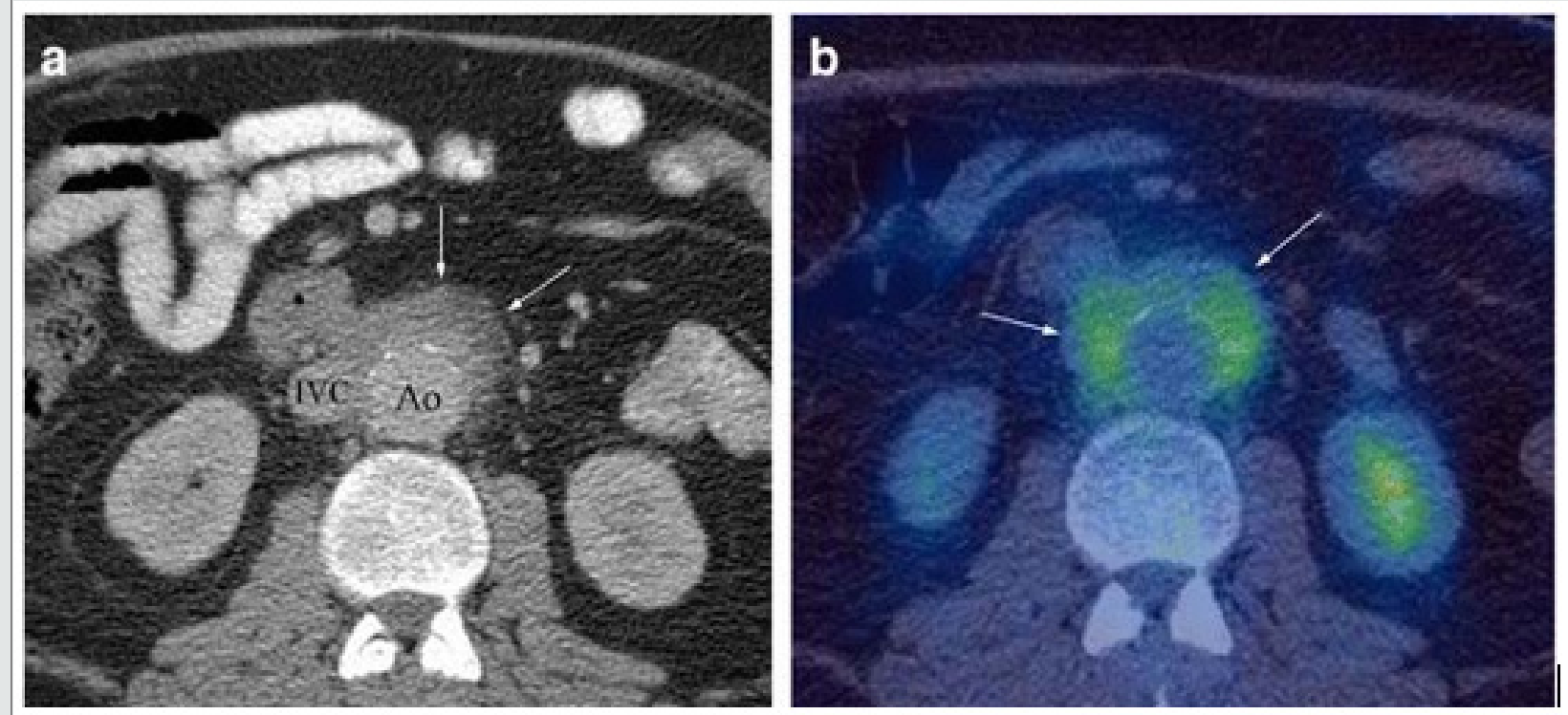

Figure 4: Example of IgG4 retroperitoneal fibrosis. 4a Axial CECT showing enhancing soft tissue deposit partly encasing the IVC and atherosclerotic aorta (arrows). b Axial FDG PET/CT showing increased FDG uptake in the abnormal retroperitoneal soft tissue (arrows). (Courtesy: Christopher Siew Wai Tanghttps://insightsimaging.springeropen.com/articles/10.1007/ s13244- 018-0618-1).

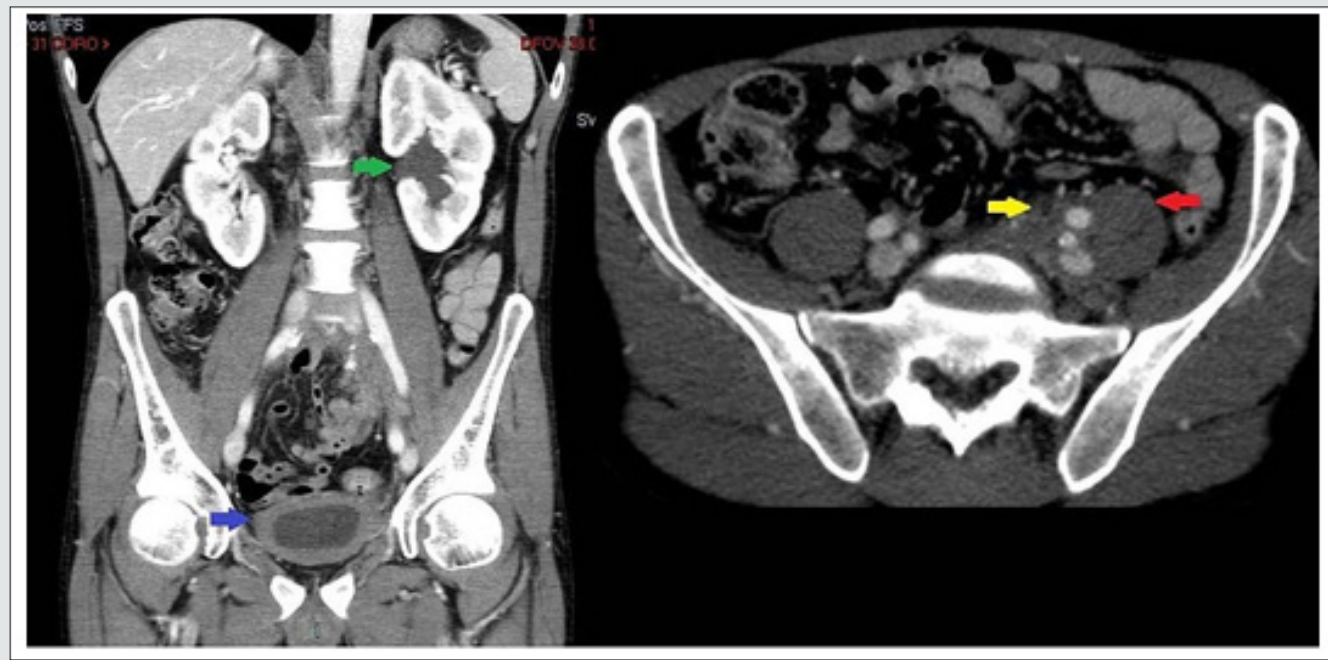

Figure 5a: Axial and 5b: coronal computerized tomography images demonstrating left-sided pelvic mass causing hydroureteronephrosis (green arrow) with diffuse bladder wall thickening (blue arrow). Asymmetric pelvic mass extending to posterior presacral region and psoas (red arrow), with ureter encased by pelvic mass (yellow arrow). Courtesy: R Bechtold, M I Shaff; PMID: 6879267; DOI: 10.1097/00007611-198308000-00023.

\section{Perianeurysmal Fibrosis}

Reports of 56 previous cases showed striking male predominance. An abdominal mass may be palpable in $45 \%$ of cases. Fibrosis is often seen in association with atherosclerosis of the aorta (Figure 6), possibly due to fibrosis as an immune response to the leakage of 'ceroid' from the atheromatous plaque into the perivascular tissues. When perianeurysmal fibrosis occurs in association with an abdominal aortic aneurysm it may encase and produce ureteric obstruction and renal function impairment. The computerized tomography scan is able to provide accurate details of aortic aneurysm, intraluminal thrombosis, calcification, periaortic inflammation, entrapped ureters and hydronephrosis (Figure 6). 


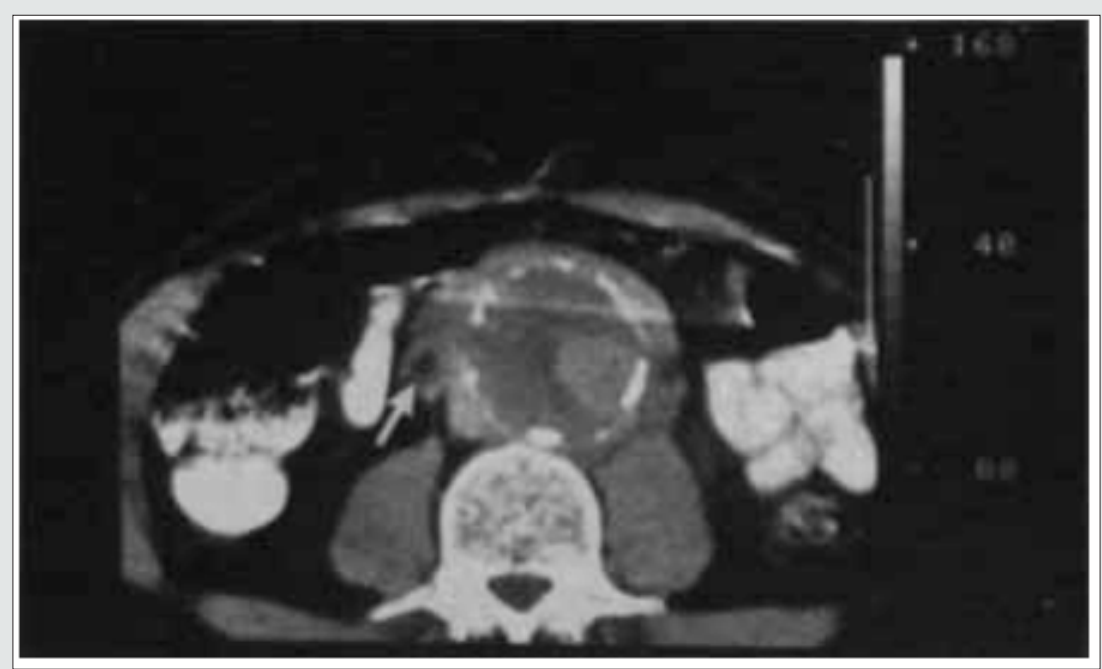

Figure 6: At L3/4 DISC SPACE level - Aorta enlarged with calcifications in the wall. The left ureter (black arrow) is encased by the periaortic aneurysmal fibrosis and the right ureter (white arrow) lies adjacent to fibrosis (partly encased). Both ureters are small, the possibility of Ureteric obstruction secondary to perianeurysmal fibrosis should be in the pre-operative evaluation of abdominal aortic aneurysms. (Courtesy: https://www.researchgate.net/ R N Gibson Alison Halliday A Mansfield).

\section{Neoplasm}

Locally invasive neoplasm in the retroperitoneum and pelvis can involve ureters by three ways: 1 . by malignant infiltration of their walls causing narrowing of their lumen (stricture) and affecting the peristalsis; 2 . by external compression by the mass itself that may encroach, encompass and compress and displace the kidneys and ureters (Figure 7a) or by the adjacent aorto-caval and common iliac lymphadenopathy around abdominal ureter and internal and external iliac nodes around the pelvic ureter (carcinoma of prostate, uterus, cervix and or colon cause lymph node enlargement or organ enlargement); 3 . by encircling and encasing the ureter as a whole. True hematogenous or lymphatic metastases to the ureter may occur from primary somewhere (Figure $7 \mathrm{~b}$ ). The primary may be in stomach, pancreas, lung, breast, neuroendocrine tumour) But it is exceedingly uncommon to find ureteral obstruction due to metastases to the ureter from distant primary tumors. Until now, only about 400 cases confirmed by post-mortem have been reported $[20,21]$.

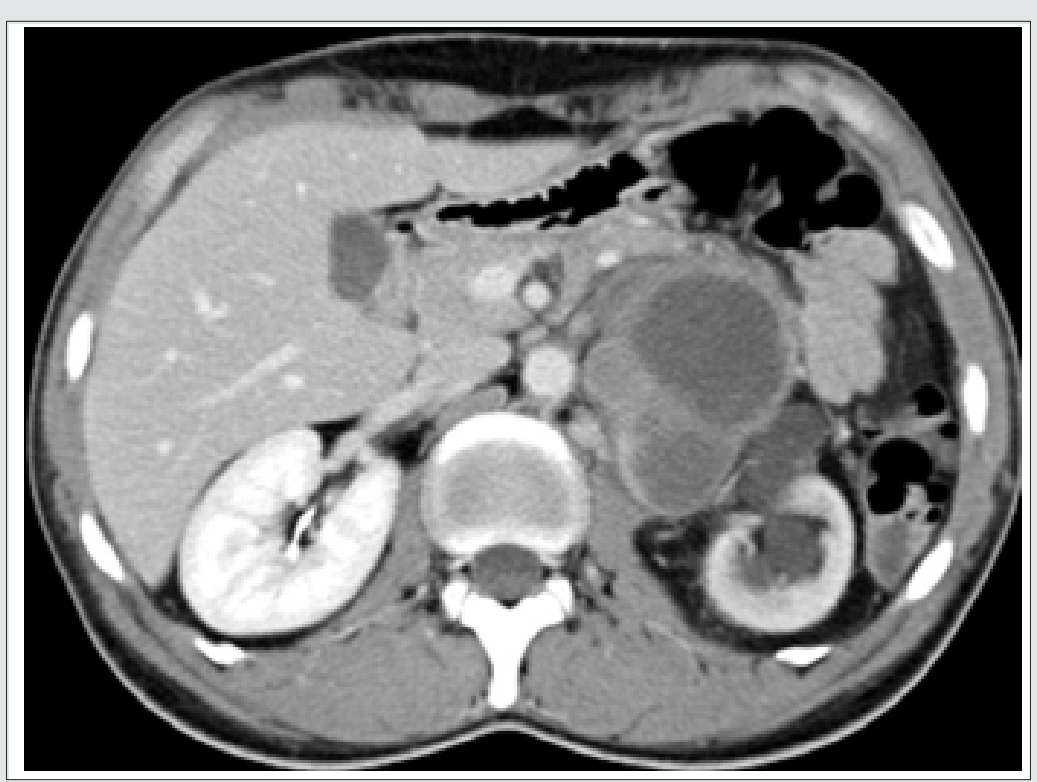

Figure 7: Retroperitoneal soft tissue sarcoma Encased and dilated left ureter (https:/ /radiopaedia.org/cases/retroperitoneal). 


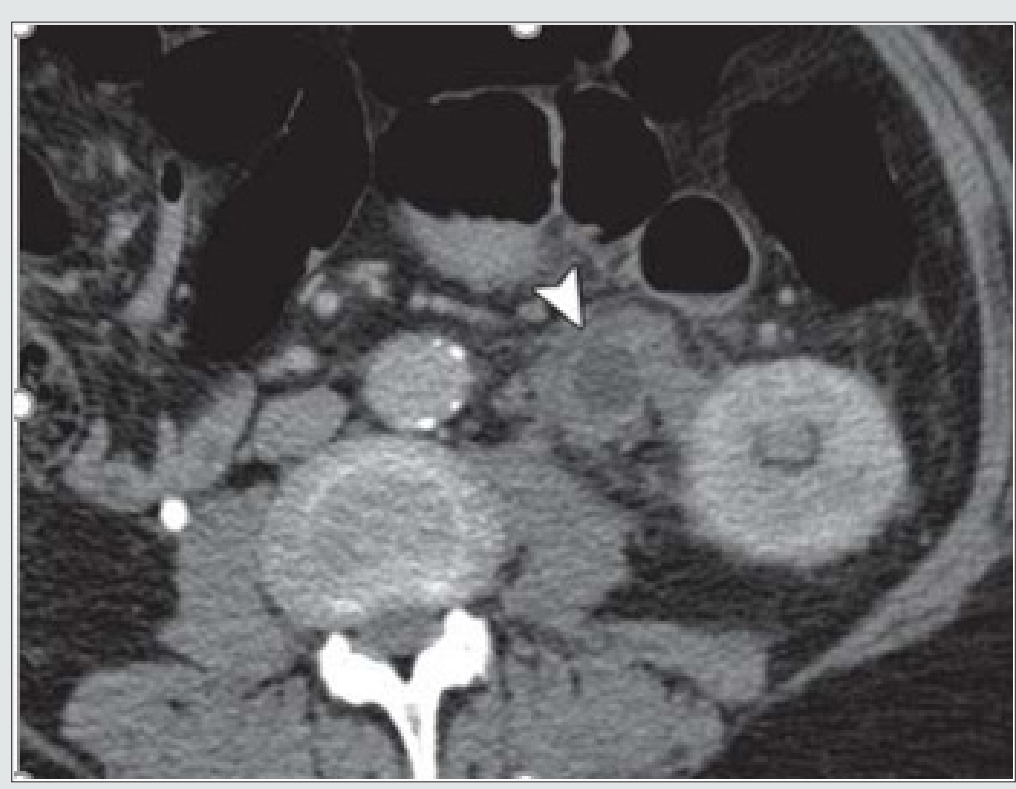

Figure 7b: Ureteral metastasis of lung cancer Encased and dilated left ureter https://cdn.amegroups.cn/journals/amepc/fil.

\section{Endometriosis}

Currently endometriosis is considered between benign and malignant status and is known as a "malignant" benign disease by experts. Its histopathologic, and molecular data suggest that endometriosis has malignant potential and is associated with ovarian cancer $[22,24]$. Although urinary tract endometriosis occurs in $\sim 1 \%$ of women mainly in women of child-bearing age with pelvic endometriosis it may cause hydronephrosis by involving the ureters secondarily by encompassing and or compressing them. Any lapse or delayed diagnosis can lead to renal failure. Ultrasound, CT and MRI (Figure 8) may be revealed and or laparoscopy may reveal the encasement of ureters.

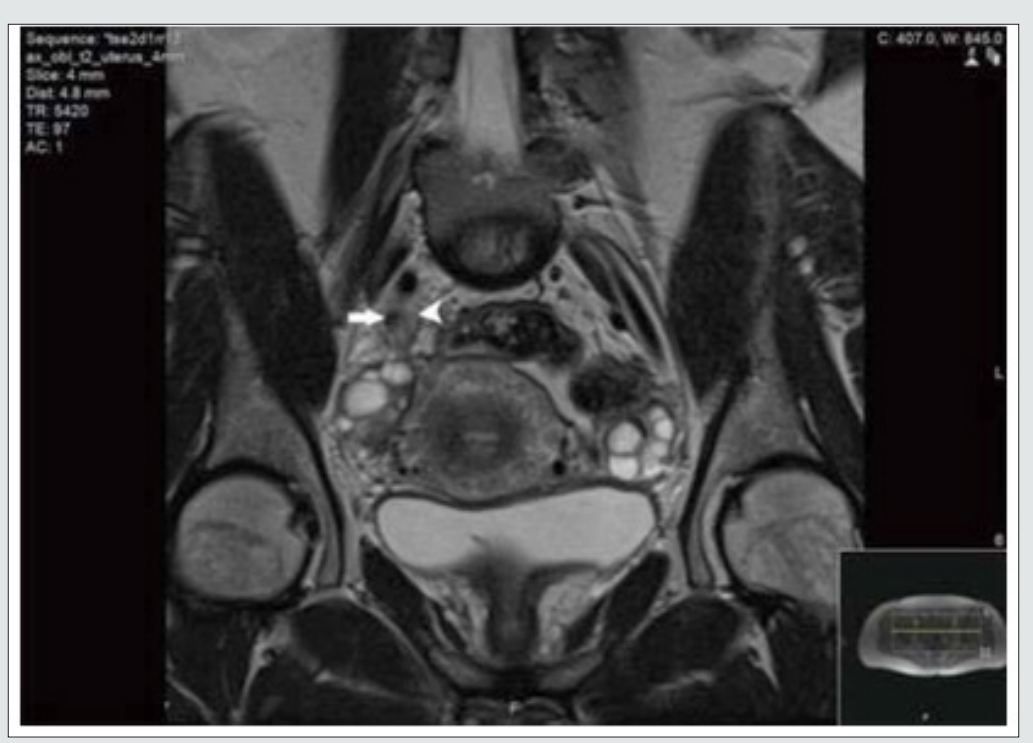

Figure 8: MRI scan of the pelvis showing abnormal tissue (arrowhead) which encases the right distal ureter (arrow). (Courtesy: Apostolos Vrettos, Maria Prasinou, Rob Frymann doi: 10.21037/qims.2016.01.02)

\section{Conclusion}

Both ureters are retroperitoneal throughout their course in the abdomen. An encompassing inflammation and or infiltrative malignant process usually cause circumferential wall thickening and an abrupt change in the diameter of the ureter resulting in dilated proximal ureter with a narrow or normal-calibred distal ureter. RPF is usually diagnosed through clinical presentation 
and imaging studies. The severity of RPF is emphasised by the fact that, in about $56 \%-100 \%$ of patients with idiopathic RPF, the fibroinflammatory tissue entraps the ureters and causes obstructive uropathy and subsequent renal failure. However, in any case biopsy should be considered to exclude malignancy. Any of the above discussed pathologies may involve the renal vessels and contribute to renal insufficiency or cause renovascular hypertension. New onset of hypertension, flank pain radiating to groin in a known case of RPF or malignancy should arise the suspicion of ureteral encasement and appropriate imaging should confirm. Failure in recognition of the encasement of ureters and its causes may lead to flawed diagnosis and irrecoverable damage to the kidneys. Early detection, corticosteroids, Radiation therapy, Retrograde ureteral stent and PCN placement, bilateral ureterolysis and resection of the aneurysm and other surgical interventions are some in the management algorithm to be chosen to broaden the treatment arena and provide encouraging results.

\section{Acknowledgement and Disclaimer}

The author would like to thank Taylor's University, Malaysia for the time granted for reviewing work. I am also grateful for the insightful comments offered by the anonymous peer reviewers of the Journal of Urology \& Nephrology studies. This paper would not have been possible without the exceptional support of my amazing partner A. Riaz Ahmed. His enthusiasm, contributions and the responses kept my work on track.

This article entitled "“'Swathed ureter, an enigma in DiagnosisA pictorial essay" is for "Research, Teaching and Learning" purpose only. The content of this is meant only as a source of valuable information for the reader; the materials contained on this do not constitute legal or other professional advice on any subject matter. Please consult with your own resources, physician or healthcare specialist regarding the suggestions and recommendations made in this to determine what may be best for your individual needs. Information and illustrations provided are taken from many (re) sources and are duly and gratefully acknowledged by quoting them as 'courtesy/Ref'. While the information contained within this article does not refer to a particular patient. Some web links within this article are solely for the convenience of reference to the reader, and the presence of such a link does not imply a responsibility for the linked site or an endorsement of the linked site, its operator, or its contents. Although the author has made every effort to ensure that the information in this book was correct at press time in regard to the subject matter covered, the publisher and the author assume no responsibility for errors, inaccuracies, omissions, or any other inconsistencies herein. The contents of this site in any form of reproduction, permanent storage, or retransmission are prohibited without the prior written consent of the quoted/referred authors and publishers.

\section{References}

1. A Schlegel PN, Chughtai B (2014) Malignant ureteral obstruction. Urology \& Nephrology Open Access Journal 1(1): 11-13.
2. Apostolos Vrettos, Maria Prasinou, Rob Frymann (2016) Ureteral endometriosis: an uncommon cause of ureteral stricture. Quantitative Imaging in Medicine and Surgery 6(2): 231-232.

3. Ali Jiwani (2013) Ureteral endometriosis: an uncommon cause of ureteral stricture.

4. Chung SY, Stein RJ, Landsittel D (2004) 15-year experience with the management of extrinsic ureteral obstruction with indwelling ureteral stents. J Urol 172(2): 592-595.

5. Chen HC, Shen SH, Wang JH (2011) Parallel second stent placement for refractory ureteral stent malfunction in malignant ureteral obstruction. J Vasc Interv Radiol 22(7): 1012-1016.

6. Gerullis H, Ecke TH, Schwartmann K (2010) Nephrocutaneous bypass in ureteral obstruction. Urology 76(2): 480-485.

7. Ganatra AM, Loughlin KR (2005) The management of malignant ureteral obstruction treated with ureteral stents. J Urol 174(6): 2125-2128.

8. Geoghegan T, Byrne AT, Benfayed W, McAuley G, Torreggiani WC (2007) Imaging and intervention of retroperitoneal fibrosis. Australas Radiol 51: 26-34.

9. Hyams ES, Shah O (2008) Malignant extrinsic ureteral obstruction: a survey of urologists and medical oncologists regarding treatment patterns and preferences. Urology 72(1): 51-56.

10. Ishioka J, Kageyama Y, Inoue M (2008) Prognostic model for predicting survival after palliative urinary diversion for ureteral obstruction: analysis of 140 cases. J Urol 180(2): 618-621.

11. Inoue D, Zen Y, Abo H (2011) Immunoglobulin G4-related periaortitis and periarteritis: CT findings in 17 patients. Radiology 261: 625-633.

12. Joshi HB, Adams S, Obadeyi 00 (2001) Nephrostomy tube or 'JJ' ureteric stent in ureteric obstruction: assessment of patient perspectives using quality-of-life survey and utility analysis. Eur Urol 39(6): 695-701.

13. Liatsikos EN, Karnabatidis D, Katsanos K (2009) Ureteral metal stents:10-year experience with malignant ureteral obstruction treatment. J Urol 182(6): 2613-2617.

14. L R I Baker, $\mathrm{r}$ Croxson, $\mathrm{n}$ Khader, $\mathrm{r} \mathrm{H}$ Reznek, $\mathrm{m}$ Al rukhaimi, et al. (1992) Rate of Development of Ureteric Obstruction in Idiopathic Retroperitoneal Fibrosis (Peri-aortitis). British Journal of Urology 69(1): 102-105.

15. Monsky WL, Molloy C, Jin B (2013) Quality-of-life assessment after palliative interventions to manage malignant ureteral obstruction. Cardiovasc Intervent Radiol 36(5): 1355-1363.

16. M Kunin, W E Goodwin (1990) The encased ureter: bullet and bodkin pattern, a reliable radiographic sign. Br J Urol 66(5): 471-474.

17. Okazaki K, Umehara H (2012) Are classification criteria for IgG4-RD now possible? The concept of IgG4-related disease and proposal of comprehensive diagnostic criteria in Japan. Int J Rheumatol 2012: 357071.

18. Raymond B Dyer, Michael Y Chen, Ronald J Zagoria (2004) Classic Signs in Uroradiology. RadioGraphics 24: suppl_1.

19. R N Gibson, Alison Halliday, A Mansfield (1988) Abdominal Aortic Aneurysm with Perianeurysmal Fibrosis: Demonstration of Ureteric Obstruction by Computerized Tomography. Australas Radiol 32(1): 149152.

20. R Bechtold, M I Shaff (1983) Pelvic lipomatosis with ureteral encasement and recurrent thrombophlebitis. South Med J 76(8): 1030-1032.

21. R Rault, W Kapoor, W Kam (1982) Perianeurysmal fibrosis and ureteric obstruction: case report and review of literature. Clin Nephrol 18(3): 159-162. 
22. Stuart G Silverman, John R Leyendecker, E Stephen Amis (2009) What Is the Current Role of CT Urography and MR Urography in the Evaluation of the Urinary Tract? Radiology 250(2).

23. Yu SH, Ryu JG, Jeong SH (2013) Predicting factors for stent failure-free survival in patients with a malignant ureteral obstruction managed with ureteral stents. Korean J Urol 54(5): 316-321.
24. Yabusaki S, Oyama Manabe N, Manabe O (2017) Characteristics of immunoglobulin G4-related aortitis/periaortitis and periarteritis on fluorodeoxyglucose positron emission tomography/computed tomography co-registered with contrast-enhanced computed tomography. EJNMMI Res 7(1): 20.

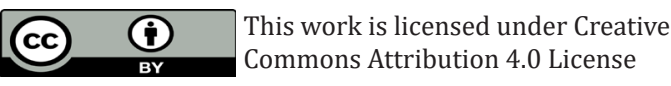

To Submit Your Article Click Here:

Submit Article

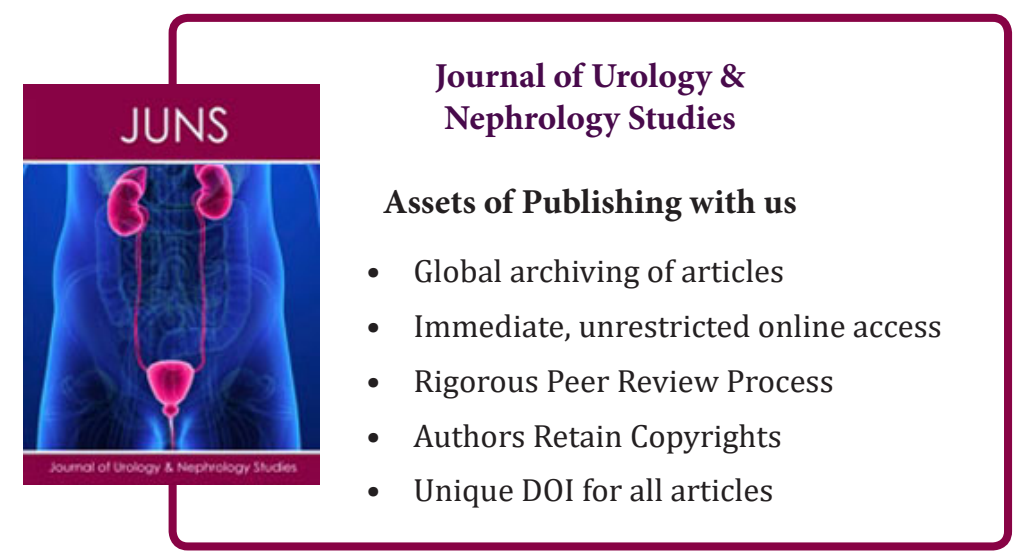

